\title{
New Breakthroughs for Liver Transplantation of Cholangiocarcinoma
}

\author{
Robert R. McMillan $^{1} \cdot$ Ashish Saharia $^{1} \cdot$ Maen Abdelrahim $^{2} \cdot$ R. Mark Ghobrial ${ }^{1}$
}

Accepted: 7 January 2021 / Published online: 2 February 2021

(C) The Author(s) 2021

\begin{abstract}
Purpose Liver transplantation (LT) has gained ground as a treatment for cholangiocarcinoma. LT has achieved an established role as curative treatment for unresectable hilar cholangiocarcinoma (hCCA), and there are emerging data supporting its use in select patients with intrahepatic cholangiocarcinoma (iCCA). This review explores the current frontiers and future directions of LT for CCA.

Recent Findings Results of retrospective studies support randomized trials of LT for resectable hCCA. Select patients with iCCA who have small tumors or favorable disease biology, as demonstrated by response to neoadjuvant therapy, have long-term survival after LT. Discoveries in the biology of iCCA show patients with FGFR and other genetic abnormalities have improved outcomes and may benefit from targeted therapies.

Summary The outcomes of LT for CCA support its increased utilization. Policies should be considered to increase accessibility of donor organs for patients with iCCA.
\end{abstract}

Keywords Cholangiocarcinoma $\cdot$ Liver transplantation $\cdot$ Mayo protocol $\cdot$ Neoadjuvant therapy

\section{Introduction}

Cholangiocarcinoma (CCA) is a malignancy of the bile duct epithelium, and it is the second most common primary liver malignancy $[1,2]$. CCA is divided into three groups by anatomic origin: intrahepatic cholangiocarcinoma (iCCA) includes lesions above the hilar junction of the bile ducts; hilar cholangiocarcinoma (hCCA) includes lesions above the cystic duct and within the bile duct hilum; and distal cholangiocarcinoma includes lesions from the ampulla of Vater to the cystic duct. These anatomic subtypes have unique disease behavior, patient outcomes, and different treatment options. Liver transplantation (LT) has become an established treatment modality for hCCA, as evidenced by UNOS awarding MELD

This article is part of the Topical Collection on Liver Transplant Oncology

Robert R. McMillan

rrmcmillan@houstonmethodist.org

$1 \quad$ Sherrie and Alan Conover Center for Liver Disease and Transplantation, J.C. Walter Jr Transplant Center, Weill Cornell Medical College, Houston Methodist Hospital, Houston, TX, USA

2 Section of GI oncology, Houston Methodist Cancer Center and Cockrell Center for Advanced Therapeutics Phase I program, Houston Methodist Research Institute, Houston, TX, USA exception points for qualified patients seeking LT. The role of LT for iCCA is evolving. After early poor results, iCCA was considered a contraindication to LT; however, recent studies have shown favorable outcomes in select patients. This review explores the current frontiers and future directions of LT for CCA.

\section{Epidemiology and Risk Factors}

Every year, 40,000 patients are diagnosed with primary liver cancers [3]. Of this group, 15-20\% have CCA [1]. The incidence of CCA has risen in the last four decades, most substantially in the iCCA subtype. From 1973 to 2012, the incidence of iCCA increased from 0.44 to 1.18 cases per 100,000 person-years - a $2.3 \%$ annual percent change (APC) - while the incidence of extrahepatic CCA increased from 0.96 to 1.02 per 100,000 - a $0.14 \%$ APC [4]. There has also been a significant rise in the mortality rate of iCCA. From 2002 to 2012, the US mortality rate increased among men from 1.42/ 100,000 person-years to $2.08 / 100,000$, and among women from $1.13 / 100,000$ person-years to $1.69 / 100,000$, increases of 46.5 and $49.6 \%$, respectively. Conversely, over the same time period, the mortality rate from extrahepatic CCA decreased for both men and women by $18 \%$ [5]. 
The reasons for the increased incidence and mortality rate of iCCA are unclear but may relate to more widespread use of high-quality cross-sectional imaging and a rise in the prevalence of risk factors [6]. Common risk factors for hCCA and iCCA include primary sclerosing cholangitis (PSC), choledochal cysts, cirrhosis, hepatitis $\mathrm{B}$, hepatitis $\mathrm{C}$, nonalcoholic steatohepatitis (NASH), diabetes, tobacco, and alcohol use. Risk factors unique to iCCA include hepatolithiasis and hemochromatosis [1, 7]. A recent meta-analysis of risk factors found cirrhosis to be the most significant risk factor for iCCA, with a combined OR of 22.92 (95\% CI $=18.24-28.79)$ [8]. Liver flukes (i.e., Clonorchis sinensis and Opisthorchis viverrini) are a common cause of cholangiocarcinoma in endemic areas such as Thailand, which has the highest rate of cholangiocarcinoma in the world [9].

\section{Clinical Presentation and Diagnosis}

The clinical presentation of CCA depends on tumor location and stage of disease. Extrahepatic CCA often results in biliary obstruction, causing jaundice, pruritis, pale stools, and dark urine. Laboratory abnormalities include elevations in liver function tests and the tumor marker CA 19-9. By contrast, iCCA is usually asymptomatic, though advanced cases can cause right upper quadrant pain and weight loss [10]. Cross-sectional imaging for hCCA may show bile duct thickening or stricture with intrahepatic biliary dilatation [11]. On imaging, iCCA is mass forming, with peritumoral biliary dilatation, surface retraction, and the lack of a definitive capsule. iCCA lesions typically show diffuse arterial uptake with continued enhancement on venous phases without washout. The hepatobiliary phases of gadolinium MRI show conspicuous tumor-liver interphase $[11,12]$.

Diagnostic criteria set forth by UNOS for patients with hCCA require either tissue biopsy or cytology showing adenocarcinoma, aneuploidy on fluorescence in situ hybridization (FISH), or the presence of a malignant-appearing stricture with an elevated CA 19-9 $\geq 100 \mathrm{U} / \mathrm{mL}$ [13]. Percutaneous biopsy for hCCA is avoided because it violates the tumor plane and renders a patient ineligible for MELD exception points. Due to the pauci-cellular, fibrous nature of hCCA tumors, obtaining a tissue diagnosis can be difficult. Brush cytology has a sensitivity of $30 \%$, which may be improved upon by using FISH, which has a sensitivity of $60 \%$, or singleoperator cholangioscopy, which has a sensitivity of $80 \%$ [14]. The diagnosis of iCCA is made with percutaneous biopsy. A liver biopsy showing adenocarcinoma requires clinical correlation, however, as metastatic adenocarcinoma from the GI tract or lung may appear histologically like iCCA [15].

\section{Surgical Resection}

Historically, surgical resection has been the preferred curative option for CCA. However, a minority of patients with hCCA and iCCA are resectable at the time of diagnosis $[16,17]$.

Resectability depends on tumor location and the size and quality of the future liver remnant (FLR). Patients with underlying liver disease need to be evaluated for portal hypertension, and an adequate FLR must be ensured to avoid postoperative liver failure. Modalities to increase the size of the FLR include portal vein embolization (PVE) and associated liver partition and portal vein ligation for staged hepatectomy (ALPPS).

Resection for hCCA involves removal of the involved bile ducts and hepatic lobe as well as the caudate lobe [18]. A multi-institutional study from 2018 affirmed caudate resection is associated with a greater likelihood of a margin-negative resection but did not find it yielded significantly improved overall survival (OS) or recurrence-free survival (RFS) [19]. Resection for iCCA includes non-anatomic resections for small, peripheral lesions and anatomic hepatectomies for larger tumors. A retrospective review from 2019 showed superior OS and RFS with anatomic resection over non-anatomic resection for patients with stage IB or II iCCA without vascular invasion [20].

Five-year survival for resection ranges from 25 to $50 \%$ for hCCA and 25-40\% for iCCA [21-25]. Both types of CCA have high recurrence rates ranging from 50 to $70 \%[23,26$, 27]. Factors associated with long-term survival after resection of hCCA include lower median pretreatment CA 19-9 levels (115 vs $34 \mathrm{U} / \mathrm{mL} ; p=0.008)$ and a lower rate of positive lymph nodes [28]. For iCCA, significant predictors of death and recurrence were age, tumor size, multiple lesions, lymph node and vascular involvement and poor tumor differentiation [27]. An analysis from 2015 showed the probability of cure after resection of iCCA was $9.7 \%$ at 10 years after surgery, while patients with small, well-differentiated single tumors had the highest probability of cure at $25.8 \%$ [29].

\section{Transplantation for Hilar Cholangiocarcinoma}

Early experience with LT for hCCA had disappointing results, with 5-year survival rates of less than $30 \%$ [30-32]. The breakthrough report of successful LT for hCCA came from the University of Nebraska in 2002, where neoadjuvant chemoradiation followed by transplantation was found to yield long-term survival [33]. This approach led to the creation of the so-called Mayo Protocol for LT of hCCA. The efficacy of this protocol was reported in seminal papers from 2005 and 2012 , with 5-year recurrence free survival of $65 \%$ [34, 35]. Given the success of this treatment approach, UNOS awards 
MELD exception points for patients who meet criteria set forth by the protocol [13].

Patient selection for the Mayo Protocol requires diagnosis of hCCA that is unresectable due to the inability to restore biliary discontinuity (e.g., bilateral segmental ductal extension) or an inadequate hepatic remnant [18]. Included in this unresectable group are patients with underlying parenchymal disease and patients with PSC, who have a high rate of multifocal CCA. Patients are excluded if they have extrahepatic disease, regional lymph node disease, or large radial tumor size $>3 \mathrm{~cm}$. Patients undergo neoadjuvant chemoradiotherapy with 5-Fluoruracil and 4500 cGy external beam radiation, followed by maintenance oral capecitabine until transplant. Some centers include brachytherapy using intraductal iridium coils to deliver an additional 2000-3000 cGy to the tumor. Lastly, patients undergo operative staging to rule out lymphatic or peritoneal disease, either as a separate operation before LT or at the time a donor liver becomes available. Patients who start the protocol with intent to treat with LT may drop out due to disease progression or other issues. In the multicenter report from 2012, 11.5\% of patients dropped out before transplant [34•].

Transplanting patients using the Mayo protocol carries with it operative challenges. The irradiated porta hepatis makes dissection difficult and can endanger vascular anastomoses. Therefore, many surgeons transect the artery, portal vein, and bile duct close to the duodenum, avoiding the most heavily irradiated part of the hilum and minimizing the risk of tumor spread [36•]. Radiation injury to the recipient structures is progressive over time, and patients transplanted for hCCA have a significantly higher rate of late hepatic artery thrombosis and portal vein complications compared with non-hCCA controls -18.9 versus $4.1 \%, p<0.001$, and 37.8 versus $8.7 \%$, $p<0.001$, respectively $[37,38]$. Operative strategies to mitigate post-radiation complications include the use of vascular conduits and Roux-en-Y biliary reconstruction to avoid using irradiated structures in the porta. Despite these technical challenges, patient survival is dictated by tumor response to neoadjuvant treatment and the presence of residual cancer on explant, not vascular or biliary complications [38, 39].

Because LT for hCCA has shown consistent favorable outcomes in selected patients with unresectable disease, some have considered expanding LT to patients with resectable disease. In a multi-institutional report from 2018, outcomes of successfully resected patients who otherwise met criteria for LT $(<3 \mathrm{~cm}$ tumors with node negative disease) were compared with unresectable patients who underwent LT. The group undergoing LT had superior OS compared with resection $(5$-year 64 versus 18\%, $p<0.001)$ [40]. A multi-center randomized trial of resection versus neoadjuvant chemoradiation followed by LT is now underway to ascertain which treatment modality is superior among resectable patients (NCT02232932).

\section{Transplantation for Intrahepatic Cholangiocarcinoma}

\section{Recent Reports with Favorable Outcomes}

As with hCCA, the first attempts at LT for patients with iCCA were met with poor outcomes. Due to a lack of patient selection and higher perioperative mortality, 5-year survival after LT rarely exceeded $30 \%[32,41-43]$. As a result, most transplant centers made iCCA a contraindication to LT. However, patients with iCCA continued to undergo LT for several reasons: (1) the presence of undiagnosed, or incidental, small iCCA lesions found on liver explant; (2) the misdiagnosis of iCCA lesions as hepatocellular carcinoma (HCC); and (3) the presence of iCCA tumor nodules in patients with separate HCC tumor nodules. Retrospective studies published in the last 5 years have examined patients transplanted for iCCA and found groups with favorable outcomes.

In a 2014 multicenter study of Spanish transplant centers and a subsequent international multicenter study from 2016, Sapisochin and coworkers examined LT patients with incidentally discovered iCCA and patients with iCCA tumors misdiagnosed as HCC [44, 45]. Patients $(n=48)$ were divided into a "very early" iCCA group if they had single tumors with diameter $\leq 2 \mathrm{~cm}(15 / 48)$ or an "advanced" group if they had either tumors $>2 \mathrm{~cm}$ or multiple tumors (33/48). "Very early" patients had favorable cumulative recurrence rates at 1,3 , and 5 years of 7 , 18 , and $18 \%$, compared with "advanced" patients with 30 , 47 , and $61 \%(p=0.01)$. One-year, 3-year, and 5-year OS among "very early" patients was also favorable at 93,84 , and $65 \%$, compared with the "advanced" group at 79,50 , and $45 \%$. Among the "very early" group, two of the five patients who died had tumor recurrence, while most of the patients in the advanced group who died had recurrence. The authors performed a second analysis of 33 patients with mixed iCCA-HCC present in separate tumor nodules [45•]. Patients were again divided up by the diameter of the iCCA tumor nodule into a "very early" group for tumors $\leq 2 \mathrm{~cm}(n=14)$ and an "advanced" group for tumors $>2 \mathrm{~cm}(n=19)$. The "very early" iCCA + HCC tumor group had significantly improved RFS and OS compared with the "advanced" group, with 5-year cumulative risk of recurrence of 23 versus $61 \%(p=0.04)$ and 5 -year survival of 84 versus $43 \%(p=0.04)$, respectively (Table 1). From their analyses, the authors concluded patients with "very early" iCCA tumors are a select group with favorable recurrence rates and survival, and they have started a prospective trial of patients with small iCCA undergoing deceased donor LT (NCT02878473).

More recent reports have shown favorable outcomes of LT for iCCA in patients with tumor diameters exceeding $2 \mathrm{~cm}$. A French multicenter retrospective study published in 2020, 
Table 1 Liver Transplantation for iCCA after 2014

\begin{tabular}{|c|c|c|c|c|}
\hline Author & Year & Number of Patients & Overall Survival & Recurrence \\
\hline \multirow[t]{2}{*}{ Sapisochin et al } & \multirow[t]{2}{*}{2014} & 29, Total & 5 -year, $45 \%$ & 5 -year cumulative risk of recurrence, $29 \%$ \\
\hline & & 8, "Very Early" group & 5 -year, $73 \%$ & No recurrences \\
\hline \multirow{6}{*}{$\begin{array}{l}\text { Sapisochin and iCCA international } \\
\text { Consortium et al }\end{array}$} & \multirow[t]{6}{*}{2014} & 48, Total, iCCA only & & \\
\hline & & 15, "Very Early" iCCA only & 5 -year, $65 \%$ & 5 -year cumulative risk of recurrence, $18 \%$ \\
\hline & & 33, "Advanced" iCCA only & 5 -year, $45 \%$ & 5 -year cumulative risk of recurrence, $61 \%$ \\
\hline & & 33, Total, iCCA + HCC & & \\
\hline & & 14, "Very Early" iCCA + HCC & 5 -year, $84 \%$ & 5 -year cumulative risk of recurrence, $23 \%$ \\
\hline & & 19, “Advanced” iCCA + HCC & 5 -year, $43 \%$ & 5 -year cumulative risk of recurrence, $61 \%$ \\
\hline \multirow[t]{2}{*}{ Facciuto et al } & \multirow[t]{2}{*}{2014} & 32 , Total & 5 -year, $57 \%$ & 5-year, disease-free survival, $44 \%$ \\
\hline & & 10, "Within Milan" group & 5 -year, $78 \%$ & 5-year, disease-free survival, $78 \%$ \\
\hline \multirow[t]{3}{*}{ De Martin et al } & \multirow[t]{3}{*}{2020} & 49 , total & 5 -year, $67 \%$ & 5-year, recurrence-free survival, $75 \%$ \\
\hline & & $25,<2 \mathrm{~cm}$ Tumor group & 5 -year, $69 \%$ & Not reported \\
\hline & & $24,2-5 \mathrm{~cm}$ tumor group & 5 -year, $65 \%$ & 5 -year, recurrence-free survival, $74 \%$ \\
\hline Lunsford et al & 2018 & 6 , total & 5 -year, $83 \%$ & 5 -year, $50 \%$ \\
\hline
\end{tabular}

compared outcomes of patients undergoing $\mathrm{LT}$ or resection for iCCA and mixed iCCA-HCC with a secondary objective to evaluate outcomes of patients with tumors diameters $>2 \mathrm{~cm}$ and less than $5 \mathrm{~cm}$ [46]. Forty-nine patients had LT for an indication of end-stage liver disease or HCC and were compared with 25 patients undergoing resection. Explant pathology after LT showed iCCA in 24 patients $(49 \%)$ and mixed iCCA-HCC in 25 patients $(51 \%)$. Thirty-three $(63 \%)$ patients had pre-LT locoregional therapy, which was most commonly TACE. Among patients who had resection, none had neoadjuvant treatment while two had adjuvant chemotherapy. Results showed overall survival at 1, 3, and 5 years was 90 , 76 , and $67 \%$ for LT patients, compared with 92,59 , and $40 \%$ for patients undergoing liver resection (LR) $(p=0.165)$. RFS at 1,3 , and 5 years was 87,79 , and $75 \%$ for LT patients, versus 69,45 , and $36 \%$ ( $p=0.004)$ for patients undergoing LR. The authors performed a subgroup analysis of LT patients with tumor size between 2 and $5 \mathrm{~cm}$ and compared these patients with those with tumor diameter $\leq 2 \mathrm{~cm}$. They found comparable 5-year OS of 65 and $69 \%(p=0.4)$ between the $2-$ $5 \mathrm{~cm}$ group and $\leq 2 \mathrm{~cm}$ group, respectively, with statistically similar RFS for the two groups $(p=0.43)$ [46]. The factor most prognostic of recurrence or death on multivariate analysis was tumor differentiation (HR 3.47 and 3.23, $p=0.001$, respectively). These results correspond to a previous report from Mt. Sinai in 2015, where patients with iCCA within Milan criteria also had favorable outcomes [47]. It is not immediately apparent why patients with larger iCCA tumors achieved comparable long-term survival in these latter studies. Study patients in each report had comparable characteristics, with similar rates of pre-LT locoregional treatment and similar proportions of incidentally discovered tumors on explant versus known tumors pre-LT.

\section{Neoadjuvant Therapy and LT for iCCA}

The role of neoadjuvant therapy in LT for iCCA was first reported by Hong and colleagues at UCLA in 2011, who performed a retrospective review of patients undergoing LT or resection. The subgroup of patients in their report who had neoadjuvant therapy followed by LT had superior outcomes, compared with patients without neoadjuvant therapy [48]. These findings were confirmed in a subsequent review of LT patients with iCCA from 1985 to 2010. In this larger series, the authors devised a predictive index for tumor recurrence and identified a low risk group of patients with 5-year RFS of 78\% [49]. The success of this experience of neoadjuvant therapy influenced our decision to embark on a trial of LT for iCCA.

In 2018, our group at the Houston Methodist Hospital, in collaboration with MD Anderson Cancer Center, published the first prospective case series of neoadjuvant therapy followed by LT for patients with iCCA [50•]. We enrolled 12 patients with iCCA and no extrahepatic disease who underwent neoadjuvant chemotherapy followed by LT (Fig. 1). Tumor size was not used as a criterion for LT eligibility, rather tumor biology, as demonstrated by stable disease for 6 months on neoadjuvant therapy, determined which patients were suitable for LT. Patients underwent cross-sectional imaging of the chest and abdomen every 3 months before LT to ensure disease stability, and suspicious findings on cross-sectional imaging were evaluated with PET-CT and biopsy, as indicated. Six patients ultimately completed therapy and had LT at a median time after diagnosis of 26 months. Among these patients, the median number of lesions was 4 , and the median cumulative tumor diameter was $10.5 \mathrm{~cm}$. Neoadjuvant therapy was platinum-based with gemcitabine and cisplatin in the first 


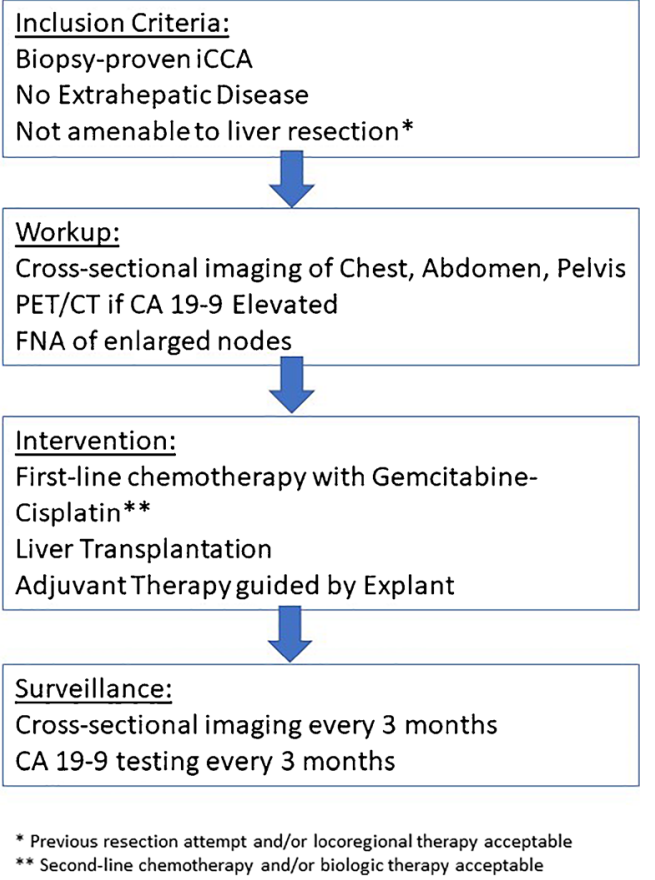

Fig. 1 Diagram of the Methodist-MD Anderson Joint Cholangiocarcinoma Collaborative Committee Prospective Trial

line, although some patients had additional lines of systemic treatment before LT [51]. Two patients who recurred after liver resection were included in the trial. Patient survival after 1,3 , and 5 years was 100,83 , and $83 \%$ and RFS at 1,3 , and 5 years was $50 \%$. Of those patients who recurred, two had lesions present before LT that, in retrospect, may have represented stable extrahepatic disease. Molecular testing was performed, and KRAS and BAPI mutations were present in two of the patients who recurred, which have been associated with an aggressive disease phenotype [52]. Since publishing our report, we have now completed LT for iCCA for 12 patients with continued favorable outcomes. Based on these positive outcomes, we believe disease stability after neoadjuvant therapy can select patients with favorable biology who will benefit from LT.

\section{Challenges and Future Directions in Liver Transplantation for Intrahepatic Cholangiocarcinoma}

Several challenges exist for more widespread adoption of LT for iCCA. First, it is difficult to obtain donor liver allografts for patients with iCCA. These patients often have a low laboratory MELD score and UNOS does not award exception points for iCCA. In our experience, we used ECD livers which otherwise would have been discarded to obtain donor organs. We believe validation of positive outcomes of LT will justify policies to facilitate deceased donor LT for iCCA. Additionally, we believe living donor liver transplantation is appropriate for patients with iCCA who have a positive response to neoadjuvant chemotherapy. The second challenge lies in patient selection. Recurrence rates after LT have ranged from 10 to $50 \%$ in recent literature. As we accrue greater experience, patient risk groups may be identified with greater precision. However, even among patients who recur, longterm survival may be possible with adjuvant chemotherapy or local therapy for recurrences.

Collaborations between transplant practitioners and oncologists are essential for advancements in complex diseases like iCCA. These specialists are founding the field of Transplant Oncology by bringing together an understanding of disease biology, prognostic factors, targeted molecular therapies, and a working knowledge of the rules and regulations of transplantation. Subgroups with less aggressive disease phenotypes will be defined who are best suited to receive the limited resource of transplantation and targeted therapies may be used as a "bridge" to transplant by maintaining disease stability, while maximizing survival after transplant.

Using next-generation technologies, genetic abnormalities (GA) in the FGFR pathway, IDH1/2, ARIDIA, BAP1, KRAS, $B R A F$, and EGFR have been discovered in iCCA [53]. Patients with $F G F R$ GA's make up an important subgroup with a prevalence of $13-17 \%$ in iCCA. This subgroup is marked by a relatively indolent course with superior progression-free survival (PFS) to first-line chemotherapy (>60 weeks) and significantly longer overall survival [54, 55]. Targeted therapies have been developed for patients in the FGFR subgroup. Two phase II trials of the FGFR kinase inhibitors BGJ398 and Pemigatinib met their primary end points with objective response rates of 14.8 and $35 \%$, respectively $[56,57]$. Other GAs suitable for targeted intervention include $I D H 1 / 2, B A P 1$, and ARIDIA. In addition, $B 7 H 1$ and $P D L 1$ have also been found to be upregulated in iCCA, which may offer avenues for immunotherapy [58]. Overall, researchers estimate as many as $70 \%$ of patients with iCCA harbor actionable mutations [59]. As we gain knowledge of iCCA subgroups and molecular therapeutics, the decision to pursue LT for patients will be informed by a more precise understanding of tumor biology.

\section{Conclusion}

Transplantation is gaining ground as a treatment for CCA. The benefits of LT for hCCA are well established, and there is an emerging body of evidence for the efficacy of LT in selected patients with iCCA. As we advance our understanding of the molecular pathogenesis of CCA, the long-term outcomes of LT for iCCA will continue to improve. With the scarcity of donor livers, advances in ECD organ transplantation and policy initiatives to increase access of LT for patients with CCA will allow more patients to benefit from the life-saving therapy of LT. 
Open Access This article is licensed under a Creative Commons Attribution 4.0 International License, which permits use, sharing, adaptation, distribution and reproduction in any medium or format, as long as you give appropriate credit to the original author(s) and the source, provide a link to the Creative Commons licence, and indicate if changes were made. The images or other third party material in this article are included in the article's Creative Commons licence, unless indicated otherwise in a credit line to the material. If material is not included in the article's Creative Commons licence and your intended use is not permitted by statutory regulation or exceeds the permitted use, you will need to obtain permission directly from the copyright holder. To view a copy of this licence, visit http://creativecommons.org/licenses/by/4.0/.

\section{References}

Papers of particular interest, published recently, have been highlighted as:

- Of importance

-• Of major importance

1. Tyson GL, El-Serag HB. Risk factors for cholangiocarcinoma. Hepatology. 2011;54(1):173-84.

2. Bergquist A, von Seth E. Epidemiology of cholangiocarcinoma. Best Pract Res Clin Gastroenterol. 2015;29(2):221-32.

3. Siegel RL, Miller KD, Jemal A. Cancer statistics, 2018. CA Cancer J Clin. 2018;68(1):7-30.

4. Saha SK, Zhu AX, Fuchs CS, Brooks GA. Forty-year trends in cholangiocarcinoma incidence in the U.S.: intrahepatic disease on the rise. Oncologist. 2016;21(5):594-9.

5. Bertuccio P, Malvezzi M, Carioli G, Hashim D, Boffetta P, ElSerag HB, et al. Global trends in mortality from intrahepatic and extrahepatic cholangiocarcinoma. J Hepatol. 2019;71(1):104-14.

6. Massarweh NN, El-Serag HB. Epidemiology of hepatocellular carcinoma and intrahepatic Cholangiocarcinoma. Cancer control : journal of the Moffitt Cancer Center. 2017;24(3): 1073274817729245.

7. Petrick JL, Yang B, Altekruse SF, Van Dyke AL, Koshiol J, Graubard BI, et al. Risk factors for intrahepatic and extrahepatic cholangiocarcinoma in the United States: a population-based study in SEER-Medicare. PLoS One. 2017;12(10):e0186643.

8. Palmer WC, Patel T. Are common factors involved in the pathogenesis of primary liver cancers? A meta-analysis of risk factors for intrahepatic cholangiocarcinoma. J Hepatol. 2012;57(1):69-76.

9. Prueksapanich P, Piyachaturawat P, Aumpansub P, Ridtitid W, Chaiteerakij R, Rerknimitr R. Liver fluke-associated biliary tract Cancer. Gut and liver. 2018;12(3):236-45.

10. Esnaola NF, Meyer JE, Karachristos A, Maranki JL, Camp ER, Denlinger CS. Evaluation and management of intrahepatic and extrahepatic cholangiocarcinoma. Cancer. 2016;122(9):1349-69.

11. Valls C, Ruiz S, Martinez L, Leiva D. Radiological diagnosis and staging of hilar cholangiocarcinoma. World journal of gastrointestinal oncology. 2013;5(7):115-26.

12. Kang Y, Lee JM, Kim SH, Han JK, Choi BI. Intrahepatic massforming cholangiocarcinoma: enhancement patterns on gadoxetic acid-enhanced MR images. Radiology. 2012;264(3):751-60.

13. Gores GJ, Gish RG, Sudan D, Rosen CB. Model for end-stage liver disease (MELD) exception for cholangiocarcinoma or biliary dysplasia. Liver Transpl. 2006;12(S3):S95-S7.

14. Rizvi S, Eaton J, Yang JD, Chandrasekhara V, Gores GJ. Emerging technologies for the diagnosis of perihilar cholangiocarcinoma. Semin Liver Dis. 2018;38(2):160-9.
15. Goodman ZD. Neoplasms of the liver. Modern pathology : an official journal of the United States and Canadian Academy of Pathology, Inc. 2007;20(Suppl 1):S49-60.

16. Poruk KE, Pawlik TM, Weiss MJ. Perioperative Management of Hilar Cholangiocarcinoma. Journal of gastrointestinal surgery : official journal of the Society for Surgery of the Alimentary Tract. 2015;19(10):1889-99.

17. Rahnemai-Azar AA, Weisbrod AB, Dillhoff M, Schmidt C, Pawlik TM. Intrahepatic cholangiocarcinoma: current management and emerging therapies. Expert review of gastroenterology \& hepatology. 2017;11(5):439-49.

18. Mansour JC, Aloia TA, Crane CH, Heimbach JK, Nagino M, Vauthey JN. Hilar cholangiocarcinoma: expert consensus statement. HPB : the official journal of the International Hepato Pancreato Biliary Association. 2015;17(8):691-9.

19. Bhutiani N, Scoggins CR, McMasters KM, Ethun CG, Poultsides GA, Pawlik TM, et al. The impact of caudate lobe resection on margin status and outcomes in patients with hilar cholangiocarcinoma: a multi-institutional analysis from the US Extrahepatic biliary malignancy consortium. Surgery. 2018;163(4):726-31.

20. Si A, Li J, Yang Z, Xia Y, Yang T, Lei Z, et al. Impact of anatomical versus non-anatomical liver resection on short- and long-term outcomes for patients with intrahepatic Cholangiocarcinoma. Ann Surg Oncol. 2019;26(6):1841-50.

21. Jarnagin WR, Fong Y, DeMatteo RP, Gonen M, Burke EC, Bodniewicz BJ, et al. Staging, resectability, and outcome in 225 patients with hilar cholangiocarcinoma. Ann Surg. 2001;234(4): 507-17 discussion 17-9.

22. Lee SG, Song GW, Hwang S, Ha TY, Moon DB, Jung DH, et al. Surgical treatment of hilar cholangiocarcinoma in the new era: the Asan experience. Journal of hepato-biliary-pancreatic sciences. 2010;17(4):476-89.

23. Rea DJ, Munoz-Juarez M, Farnell MB, Donohue JH, Que FG, Crownhart B, et al. Major hepatic resection for hilar cholangiocarcinoma: analysis of 46 patients. Archives of surgery (Chicago, Ill : 1960). 2004;139(5):514-23; discussion 23-5.

24. Hyder O, Marques H, Pulitano C, Marsh JW, Alexandrescu S, Bauer TW, et al. A nomogram to predict long-term survival after resection for intrahepatic cholangiocarcinoma: an eastern and Western experience. JAMA surgery. 2014;149(5):432-8.

25. Patel $\mathrm{T}$. Increasing incidence and mortality of primary intrahepatic cholangiocarcinoma in the United States. Hepatology. 2001;33(6): 1353-7.

26. Park SW, Park YS, Chung JB, Kang JK, Kim KS, Choi JS, et al. Patterns and relevant factors of tumor recurrence for extrahepatic bile duct carcinoma after radical resection. Hepato-gastroenterology. 2004;51(60):1612-8.

27. Mavros MN, Economopoulos KP, Alexiou VG, Pawlik TM. Treatment and prognosis for patients with intrahepatic Cholangiocarcinoma: systematic review and meta-analysis. JAMA surgery. 2014;149(6):565-74.

28. Tran TB, Ethun CG, Pawlik TM, Schmidt C, Beal EW, Fields RC, et al. Actual 5-year survivors after surgical resection of Hilar Cholangiocarcinoma. Ann Surg Oncol. 2019;26(2):611-8.

29. Spolverato G, Vitale A, Cucchetti A, Popescu I, Marques HP, Aldrighetti L, et al. Can hepatic resection provide a long-term cure for patients with intrahepatic cholangiocarcinoma? Cancer. 2015;121(22):3998-4006.

30. Ringe B, Wittekind C, Bechstein WO, Bunzendahl H, Pichlmayr R. The role of liver transplantation in hepatobiliary malignancy. A retrospective analysis of 95 patients with particular regard to tumor stage and recurrence. Ann Surg. 1989;209(1):88-98.

31. Meyer CG, Penn I, James L. Liver transplantation for cholangiocarcinoma: results in 207 patients. Transplantation. 2000;69(8): $1633-7$. 
32. Robles R, Figueras J, Turrión VS, Margarit C, Moya A, Varo E, et al. Spanish experience in liver transplantation for hilar and peripheral cholangiocarcinoma. Ann Surg. 2004;239(2):265-71.

33. Sudan D, DeRoover A, Chinnakotla S, Fox I, Shaw B Jr, McCashland T, et al. Radiochemotherapy and transplantation allow long-term survival for nonresectable hilar cholangiocarcinoma. Am J Transplant Off J Am Soc Transplant Am Soc Transplant Surg. 2002;2(8):774-9.

34. Darwish Murad S, Kim WR, Harnois DM, Douglas DD, Burton J, Kulik LM, et al. Efficacy of neoadjuvant chemoradiation, followed by liver transplantation, for perihilar cholangiocarcinoma at 12 US centers. Gastroenterology. 2012;143(1):88-98.e3; quiz e14.This study is the foundational multicenter report supporting LT for hCCA.

35. Rea DJ, Heimbach JK, Rosen CB, Haddock MG, Alberts SR, Kremers WK, et al. Liver transplantation with neoadjuvant chemoradiation is more effective than resection for hilar cholangiocarcinoma. Ann Surg. 2005;242(3):451-8 discussion 8-61.

36. Zamora-Valdes D, Heimbach JK. Liver transplant for Cholangiocarcinoma. Gastroenterol Clin N Am. 2018;47(2):26780 This recent review details the Mayo Clinic approach of LT for hCCA and also reviews LT for iCCA.

37. Mantel HT, Rosen CB, Heimbach JK, Nyberg SL, Ishitani MB, Andrews JC, et al. Vascular complications after orthotopic liver transplantation after neoadjuvant therapy for hilar cholangiocarcinoma. Liver transplantation : official publication of the American Association for the Study of Liver Diseases and the International Liver Transplantation Society. 2007;13(10):1372-81.

38. Tan EK, Rosen CB, Heimbach JK, Gores GJ, Zamora-Valdes D, Taner T. Living donor liver transplantation for Perihilar Cholangiocarcinoma: outcomes and complications. J Am Coll Surg. 2020.

39. Lehrke HD, Heimbach JK, Wu TT, Jenkins SM, Gores GJ, Rosen $\mathrm{CB}$, et al. Prognostic significance of the histologic response of Perihilar Cholangiocarcinoma to preoperative Neoadjuvant Chemoradiation in liver explants. Am J Surg Pathol. 2016;40(4): 510-8.

40. Ethun CG, Lopez-Aguiar AG, Anderson DJ, Adams AB, Fields RC, Doyle MB, et al. Transplantation versus resection for Hilar Cholangiocarcinoma: an argument for shifting treatment paradigms for Resectable disease. Ann Surg. 2018;267(5):797-805.

41. Goldstein RM, Stone M, Tillery GW, Senzer N, Levy M, Husberg $\mathrm{BS}$, et al. Is liver transplantation indicated for cholangiocarcinoma? Am J Surg. 1993;166(6):768-71 discussion 71-2.

42. Pascher A, Jonas S, Neuhaus P. Intrahepatic cholangiocarcinoma: indication for transplantation. J Hepato-Biliary-Pancreat Surg. 2003;10(4):282-7.

43. Shimoda M, Farmer DG, Colquhoun SD, Rosove M, Ghobrial RM, Yersiz H, et al. Liver transplantation for cholangiocellular carcinoma: analysis of a single-center experience and review of the literature. Liver transplantation : official publication of the American Association for the Study of Liver Diseases and the International Liver Transplantation Society. 2001;7(12):1023-33.

44. Sapisochin G. Rodríguez de lope C, Gastaca M, Ortiz de Urbina J, Suarez MA, Santoyo J, et al. "very early" intrahepatic cholangiocarcinoma in cirrhotic patients: should liver transplantation be reconsidered in these patients? Am J Transplant Off J Am Soc Transplant Am Soc Transplant Surg. 2014;14(3):660-7.

45. Sapisochin G, Facciuto M, Rubbia-Brandt L, Marti J, Mehta N, Yao FY, et al. Liver transplantation for "very early" intrahepatic cholangiocarcinoma: international retrospective study supporting a prospective assessment. Hepatology. 2016;64(4):1178-88 This multicenter retrospective study defines a population of patients with iCCA who underwent $L T$ with favorable long term outcomes.

46. De Martin E, Rayar M, Golse N, Dupeux M, Gelli M, Gnemmi V, et al. Analysis of liver resection versus liver transplantation on outcome of small intrahepatic cholangiocarcinoma and combined hepatocellular-Cholangiocarcinoma in the setting of cirrhosis. Liver transplantation : official publication of the American Association for the Study of Liver Diseases and the International Liver Transplantation Society. 2020.

47. Facciuto ME, Singh MK, Lubezky N, Selim MA, Robinson D, Kim-Schluger L, et al. Tumors with intrahepatic bile duct differentiation in cirrhosis: implications on outcomes after liver transplantation. Transplantation. 2015;99(1):151-7.

48. Hong JC, Jones CM, Duffy JP, Petrowsky H, Farmer DG, French S, et al. Comparative analysis of resection and liver transplantation for intrahepatic and hilar cholangiocarcinoma: a 24-year experience in a single center. Archives of surgery (Chicago, Ill : 1960). 2011;146(6):683-9.

49. Hong JC, Petrowsky H, Kaldas FM, Farmer DG, Durazo FA, Finn RS, et al. Predictive index for tumor recurrence after liver transplantation for locally advanced intrahepatic and hilar cholangiocarcinoma. J Am Coll Surg. 2011;212(4):514-20 discussion 20-1.

50. Lunsford KE, Javle M, Heyne K, Shroff RT, Abdel-Wahab R, Gupta N, et al. Liver transplantation for locally advanced intrahepatic cholangiocarcinoma treated with neoadjuvant therapy: a prospective case-series. The lancet Gastroenterology \& hepatology. 2018;3(5):337-48 This prospective case series reports favorable outcomes of neoadjuvant therapy followed by LT for selected patients with iCCA.

51. Valle J, Wasan H, Palmer DH, Cunningham D, Anthoney A, Maraveyas A, et al. Cisplatin plus gemcitabine versus gemcitabine for biliary tract cancer. N Engl J Med. 2010;362(14):1273-81.

52. Al-Shamsi HO, Anand D, Shroff RT, Jain A, Zuo M, Conrad C, et al. BRCA-associated protein 1 mutant cholangiocarcinoma: an aggressive disease subtype. Journal of gastrointestinal oncology. 2016;7(4):556-61.

53. Moeini A, Sia D, Bardeesy N, Mazzaferro V, Llovet JM. Molecular pathogenesis and targeted therapies for intrahepatic Cholangiocarcinoma. Clinical cancer research : an official journal of the American Association for Cancer Research. 2016;22(2):291300 .

54. Graham RP, Barr Fritcher EG, Pestova E, Schulz J, Sitailo LA, Vasmatzis G, et al. Fibroblast growth factor receptor 2 translocations in intrahepatic cholangiocarcinoma. Hum Pathol. 2014;45(8): 1630-8.

55. Churi CR, Shroff R, Wang Y, Rashid A, Kang HC, Weatherly J, et al. Mutation profiling in cholangiocarcinoma: prognostic and therapeutic implications. PLoS One. 2014;9(12):e115383.

56. Javle M, Lowery M, Shroff RT, Weiss KH, Springfeld C, Borad MJ, et al. Phase II study of BGJ398 in patients with FGFR-altered advanced Cholangiocarcinoma. Journal of clinical oncology : official journal of the American Society of Clinical Oncology. 2018;36(3):276-82.

57. Abou-Alfa GK, Sahai V, Hollebecque A, Vaccaro G, Melisi D, AlRajabi R, et al. Pemigatinib for previously treated, locally advanced or metastatic cholangiocarcinoma: a multicentre, open-label, phase 2 study. The Lancet Oncology. 2020;21(5):671-84.

58. Ye Y, Zhou L, Xie X, Jiang G, Xie H, Zheng S. Interaction of B7$\mathrm{H} 1$ on intrahepatic cholangiocarcinoma cells with PD-1 on tumorinfiltrating $\mathrm{T}$ cells as a mechanism of immune evasion. J Surg Oncol. 2009;100(6):500-4.

59. Sia D, Villanueva A, Friedman SL, Llovet JM. Liver Cancer cell of origin, molecular class, and effects on patient prognosis. Gastroenterology. 2017;152(4):745-61.

Publisher's Note Springer Nature remains neutral with regard to jurisdictional claims in published maps and institutional affiliations. 